\title{
ASYMPTOTIC ANALYSIS OF POWERS OF MATRICES
}

\author{
DIEGO DOMINICI
}

Received 13 September 2005; Revised 28 May 2006; Accepted 30 May 2006

We analyze the representation of $A^{n}$ as a linear combination of $A^{j}, 0 \leq j \leq k-1$, where $A$ is a $k \times k$ matrix. We obtain a first-order asymptotic approximation of $A^{n}$ as $n \rightarrow \infty$, without imposing any special conditions on $A$. We give some examples showing the application of our results.

Copyright (c) 2006 Hindawi Publishing Corporation. All rights reserved.

\section{Introduction}

In a recent article [1], Abu-Saris and Ahmad showed how to compute the powers of a matrix without having to compute its eigenvalues. Their main result was the following.

THeorem 1.1. If $A$ is a $k \times k$ matrix with characteristic polynomial

$$
P(x)=x^{k}+\sum_{j=0}^{k-1} a_{j} x^{j},
$$

then

$$
A^{n}=\sum_{j=0}^{k-1} b_{j}(n) A^{j}, \quad n \geq k,
$$

where

$$
\begin{array}{ll}
b_{j}(k)=-a_{j}, \quad 0 \leq j \leq k-1, & b_{-1}(n)=0, \quad n \geq k, \\
b_{j}(n+1)=b_{j-1}(n)-a_{j} b_{k-1}(n), & n \geq k, 0 \leq j \leq k-1 .
\end{array}
$$

The purpose of this paper is to find an asymptotic representation for the numbers $b_{j}(n)$ as $n \rightarrow \infty$, which using (1.2) will give an asymptotic representation of $A^{n}$ for large $n$. Since the coefficients $b_{j}(n)$ depend only on $P(x)$, our estimates will be valid for similar matrices. 
The asymptotic behavior of powers of matrices has been considered before by other authors. In $[6,7]$, Gautschi computed upper bounds for $A^{n}$ and $\left\|A^{n}\right\|$, where $\|A\|$ is a norm of $A$. Estimates of $\left\|A^{n}\right\|$ were also studied in $[2,3,12,15,16]$.

In [5], Friedland and Schneider considered the matrix

$$
B^{(m)}=A^{m}\left(I+\cdots+A^{q-1}\right), \quad m \geq 1,
$$

where $A$ is a nonnegative matrix and $q$ is a certain positive integer. They proved a theorem on the growth of $B^{(m)}$ under the assumption that the spectral radius of $A$ is equal to one. Powers of nonnegative matrices were also analyzed by Lindqvist [9]. Rothblum [11] obtained Cesaro asymptotic expansions of $\sum_{i=0}^{N} A^{i}$, where $A$ is a complex matrix with spectral radius less than or equal to one.

This paper is organized as follows: in Section 2 we find an integral representation for the exponential generating function $G_{j}(z)$ of the coefficients $b_{j}(n)$. We obtain exact formulas for $G_{j}(z)$ and $b_{j}(n)$ in the special case of the matrix $A$ having $k$ distinct eigenvalues. We conclude the section with some examples.

In Section 3 we give an exact representation and a first-order asymptotic approximation for $b_{j}(n)$, as $n \rightarrow \infty$. We consider the cases of simple and multiple eigenvalues. Our formulas are relatively easy to implement and offer very accurate estimates of $b_{j}(n)$, and therefore of $A^{n}$, for large $n$. We present some examples for different cases of $P(x)$.

\section{Generating function}

In this section, we will find an exponential generating function for the coefficients $b_{j}(n)$. First, let us define the spectral radius $\rho(A)$ of the matrix $A$ by

$$
\rho(A)=\max \{|\lambda| \mid P(\lambda)=0\} .
$$

Theorem 2.1. Let $G_{j}(z)$ be defined by

$$
G_{j}(z)=\sum_{n \geq 0} b_{j}(n+k) \frac{z^{n}}{n !} .
$$

Then,

$$
G_{j}(z)=-\frac{1}{2 \pi \mathrm{i}} \int_{c-\mathrm{i} \infty}^{c+\mathrm{i} \infty} s^{k-j-1} \frac{p_{j}(s)}{P(s)} e^{z s} d s
$$

where $c>\rho(A)$ and

$$
p_{j}(s)=\sum_{l=0}^{j} a_{l} s^{l}, \quad 0 \leq j \leq k-1,
$$

$p_{-1}(s)=0$, and $P(s)$ is the characteristic polynomial of $A$ defined in (1.1).

Proof. If we use (1.3) in (2.2), we obtain

$$
G_{j}^{\prime}=G_{j-1}-a_{j} G_{k-1}, \quad G_{j}(0)=-a_{j}, \quad 0 \leq j \leq k-1,
$$


with $G_{-1}(z)=0$. Since (2.5) is a system of first order, linear ODEs with constant coefficients, we have [4]

$$
G_{j}(z)=\sum_{l=0}^{k-1} q_{l}(z) \exp \left(\mu_{l} z\right),
$$

for some polynomials $q_{l}(z)$ and complex numbers $\mu_{l}$.

Taking the Laplace transform of $G_{j}(z)$,

$$
L_{j}(s)=\int_{0}^{\infty} G_{j}(z) e^{-z s} d z
$$

(which by (2.6) is well defined) in (2.5), we get

$$
s L_{j}+a_{j}=L_{j-1}-a_{j} L_{k-1}, \quad 0 \leq j \leq k-1,
$$

and $L_{-1}(z)=0$.

The system of linear equations (2.8) has a unique solution given by

$$
L_{j}(s)=-s^{k-j-1} \frac{p_{j}(s)}{P(s)}, \quad 0 \leq j \leq k-1
$$

where $p_{j}(s)$ is defined in (2.4). Inverting the Laplace transform in (2.9), the theorem follows.

Remark 2.2. Since

$$
\lim _{|s| \rightarrow \infty} L_{j}(s)=0, \quad 0 \leq j \leq k-1,
$$

we can replace the Bromwich contour in (2.3) with a circle $\mathscr{C}$ of radius $R>\rho(A)$ positively oriented (i.e., counterclockwise), centered at the origin [8]

$$
G_{j}(z)=-\frac{1}{2 \pi \mathrm{i}} \int_{\mathscr{C}} s^{k-j-1} \frac{p_{j}(s)}{P(s)} e^{z s} d s
$$

Corollary 2.3. If

$$
P(s)=\left(s-\lambda_{1}\right)\left(s-\lambda_{2}\right) \cdots\left(s-\lambda_{k}\right),
$$

where the eigenvalues $\lambda_{i}$ are all distinct, then

$$
\begin{gathered}
G_{j}(z)=-\sum_{l=1}^{k}\left(\lambda_{l}\right)^{k-j-1} \frac{p_{j}\left(\lambda_{l}\right)}{P^{\prime}\left(\lambda_{l}\right)} \exp \left(\lambda_{l} z\right), \\
b_{j}(n)=-\sum_{l=1}^{k}\left(\lambda_{l}\right)^{n-j-1} \frac{p_{j}\left(\lambda_{l}\right)}{P^{\prime}\left(\lambda_{l}\right)} .
\end{gathered}
$$


4 Asymptotic analysis of powers of matrices

Proof. Applying the residue theorem to (2.11), we obtain

$$
G_{j}(z)=-\sum_{P(\lambda)=0} \operatorname{Re} s\left[s^{k-j-1} \frac{p_{j}(s)}{P(s)} e^{z s} ; \lambda\right]
$$

which in turn gives (2.13) after computing

$$
\begin{aligned}
\operatorname{Re} s\left[s^{k-j-1} \frac{p_{j}(s)}{P(s)} e^{z s} ; \lambda_{l}\right] & =\lim _{s \rightarrow \lambda_{l}} s^{k-j-1} p_{j}(s) e^{z s} \frac{\left(s-\lambda_{l}\right)}{P(s)} \\
& =\left(\lambda_{l}\right)^{k-j-1} p_{j}\left(\lambda_{l}\right) \exp \left(\lambda_{l} z\right) \lim _{s \rightarrow \lambda_{l}} \frac{\left(s-\lambda_{l}\right)}{P(s)} \\
& =\left(\lambda_{l}\right)^{k-j-1} p_{j}\left(\lambda_{l}\right) \exp \left(\lambda_{l} z\right) \frac{1}{P^{\prime}\left(\lambda_{l}\right)},
\end{aligned}
$$

where in the last step we have used L'Hopital's theorem.

Writing (2.13) as

$$
G_{j}(z)=-\sum_{l=1}^{k}\left(\lambda_{l}\right)^{k-j-1} \frac{p_{j}\left(\lambda_{l}\right)}{P^{\prime}\left(\lambda_{l}\right)} \sum_{n \geq 0}\left(\lambda_{l}\right)^{n} \frac{z^{n}}{n !}
$$

and changing the order of summation, we have

$$
G_{j}(z)=\sum_{n \geq 0}\left[-\sum_{l=1}^{k}\left(\lambda_{l}\right)^{n+k-j-1} \frac{p_{j}\left(\lambda_{l}\right)}{P^{\prime}\left(\lambda_{l}\right)}\right] \frac{z^{n}}{n !},
$$

which implies that

$$
b_{j}(n+k)=-\sum_{l=1}^{k}\left(\lambda_{l}\right)^{n+k-j-1} \frac{p_{j}\left(\lambda_{l}\right)}{P^{\prime}\left(\lambda_{l}\right)} .
$$

Example 2.4. In [1] the authors considered the following examples.

(1) $P(x)=x^{3}-7 x^{2}+16 x-12=(x-2)^{2}(x-3)$.

Using (2.9) we have

$$
\begin{aligned}
& L_{0}(s)=\frac{12 s^{2}}{(s-2)^{2}(s-3)}, \\
& L_{1}(s)=\frac{-16 s^{2}+12 s}{(s-2)^{2}(s-3)}, \\
& L_{2}(s)=\frac{7 s^{2}-16 s+12}{(s-2)^{2}(s-3)},
\end{aligned}
$$

and after inverting, we obtain

$$
\begin{gathered}
G_{0}(z)=-12(8+4 z) e^{2 z}+108 e^{3 z} \\
G_{1}(z)=4(23+10 z) e^{2 z}-108 e^{3 z} \\
G_{2}(z)=-4(5+2 z) e^{2 z}+27 e^{3 z}
\end{gathered}
$$


Expanding in series, we get

$$
G_{0}(z)=-96 \sum_{n \geq 0} 2^{n} \frac{z^{n}}{n !}-48 \sum_{n \geq 0} 2^{n-1} n \frac{z^{n}}{n !}+108 \sum_{n \geq 0} 3^{n} \frac{z^{n}}{n !}
$$

and from (2.2), we conclude that

$$
b_{0}(n+3)=-96 \times 2^{n}-48 \times 2^{n-1} n+108 \times 3^{n}
$$

or

$$
\begin{aligned}
b_{0}(n) & =-96 \times 2^{n-3}-48 \times 2^{n-4}(n-3)+108 \times 3^{n-3} \\
& =-3(1+n) \times 2^{n}+4 \times 3^{n} .
\end{aligned}
$$

Similar calculations give

$$
\begin{aligned}
& b_{1}(n)=\left(4+\frac{5}{2} n\right) \times 2^{n}-4 \times 3^{n} \\
& b_{2}(n)=-\left(1+\frac{1}{2} n\right) \times 2^{n}+3^{n}
\end{aligned}
$$

in agreement with the results shown in [1].

(2) $P(x)=x^{3}-5 x^{2}+6 x=x(x-2)(x-3)$.

We can apply (2.14) directly and obtain

$$
\begin{gathered}
b_{0}(n)=0 \\
b_{1}(n)=\frac{3}{2} \times 2^{n}-\frac{2}{3} \times 3^{n}, \\
b_{2}(n)=-\frac{1}{2} \times 2^{n}+\frac{1}{3} \times 3^{n} .
\end{gathered}
$$

(3) $P(x)=x^{5}-5 x^{4}+10 x^{3}-20 x^{2}-15 x-4=(x-4)\left(x^{4}-x^{3}+6 x^{2}+4 x+1\right)$.

Although (as the authors noted) MAPLE is unable to compute the zeros of $P(x)$ exactly, it can provide us with very accurate numerical approximations

$$
\begin{gathered}
\lambda_{1}=4, \\
\lambda_{2}=0.8090169944+2.489898285 i, \\
\lambda_{3}=0.8090169944-2.489898285 i, \\
\lambda_{4}=-0.3090169944+0.2245139883 i \\
\lambda_{5}=-0.3090169944-0.2245139883 i
\end{gathered}
$$


6 Asymptotic analysis of powers of matrices

On the other hand, if Mathematica is used (thanks to one of the anonymous referees for bringing this point to our attention), the roots can be determined exactly:

$$
\begin{gathered}
\lambda_{1}=4 \\
\lambda_{2,3}=\frac{1+\sqrt{5}}{4} \pm \frac{\mathrm{i}}{2 \sqrt{2}} \sqrt{25+11 \sqrt{5}} \\
\lambda_{4,5}=\frac{1-\sqrt{5}}{4} \pm \frac{\mathrm{i}}{2 \sqrt{2}} \sqrt{25-11 \sqrt{5}}
\end{gathered}
$$

Using (2.27) or (2.28) in (2.14), we get

$$
b_{j}(n)=\frac{C_{j}}{305} 4^{n}-\sum_{l=2}^{5}\left(\lambda_{l}\right)^{n-j-1} \frac{p_{j}\left(\lambda_{l}\right)}{P^{\prime}\left(\lambda_{l}\right)}
$$

with

$$
C_{0}=1, \quad C_{1}=4, \quad C_{2}=6, \quad C_{3}=-1, \quad C_{4}=1 .
$$

Note that for $0 \leq j \leq 4$, we have

$$
\sum_{l=2}^{5}\left(\lambda_{l}\right)^{n-j-1} \frac{p_{j}\left(\lambda_{l}\right)}{P^{\prime}\left(\lambda_{l}\right)}=O\left(\left|\lambda_{2}\right|^{n}\right)=O\left(2.618^{n}\right)
$$

as $n \rightarrow \infty$.

Example 2.5. Let $A$ be the matrix

$$
A=\left(\begin{array}{cc}
1 & 2 \\
-1 & -1
\end{array}\right)
$$

with characteristic polynomial

$$
P(x)=x^{2}+1=(x-\mathrm{i})(x+\mathrm{i}) .
$$

Using (2.14), we have

$$
\begin{aligned}
& b_{0}(n)=\cos \left(\frac{\pi}{2} n\right), \\
& b_{1}(n)=\sin \left(\frac{\pi}{2} n\right),
\end{aligned}
$$

and from (1.2) we get

$$
A^{n}=\left(\begin{array}{cc}
\cos \left(\frac{\pi}{2} n\right)+\sin \left(\frac{\pi}{2} n\right) & 2 \sin \left(\frac{\pi}{2} n\right) \\
-\sin \left(\frac{\pi}{2} n\right) & \cos \left(\frac{\pi}{2} n\right)-\sin \left(\frac{\pi}{2} n\right)
\end{array}\right) .
$$


In particular, we have

$$
A^{n}= \begin{cases}I, & n \equiv 0(4), \\ A, & n \equiv 1(4), \\ -I, & n \equiv 2(4), \\ -A, & n \equiv 3(4),\end{cases}
$$

where $I$ denotes the identity matrix.

Example 2.6. This example appeared in [10]. Let $A$ be the matrix

$$
A=\left(\begin{array}{ll}
1 & 1 \\
1 & 0
\end{array}\right)
$$

with characteristic polynomial

$$
P(x)=x^{2}-x-1=(x-\alpha)(x-\beta),
$$

where

$$
\alpha=\frac{1}{2}(1+\sqrt{5}), \quad \beta=\frac{1}{2}(1-\sqrt{5}) .
$$

Then, from (2.14), we have

$$
\begin{gathered}
b_{0}(n)=\frac{1}{\sqrt{5}}\left(\alpha^{n-1}-\beta^{n-1}\right)=f_{n-1}, \\
b_{1}(n)=\frac{1}{\sqrt{5}}\left(\alpha^{n}-\beta^{n}\right)=f_{n},
\end{gathered}
$$

where $f_{n}$ is the $n$th Fibonacci number. Thus,

$$
A^{n}=\left(\begin{array}{cc}
f_{n}+f_{n-1} & f_{n} \\
f_{n} & f_{n-1}
\end{array}\right)=\left(\begin{array}{cc}
f_{n+1} & f_{n} \\
f_{n} & f_{n-1}
\end{array}\right) .
$$

\section{Asymptotic analysis}

We begin by finding an integral representation of the coefficients $b_{j}(n)$.

Lemma 3.1. The numbers $b_{j}(n)$ can be represented as

$$
b_{j}(n)=-\frac{1}{2 \pi \mathrm{i}} \int_{\mathscr{C}} s^{n-j-1} \frac{p_{j}(s)}{P(s)} d s,
$$

where $\mathscr{C}$ is a circle of radius $R>\rho(A)$ positively oriented centered at the origin, and the polynomials $p_{j}(s)$ were defined in (2.4).

Proof. Since the power series

$$
e^{z s}=\sum_{n \geq 0} s^{n} \frac{z^{n}}{n !}
$$


8 Asymptotic analysis of powers of matrices

converges uniformly on $|s| \leq R$, we can interchange integration and summation [13] in (2.11) and obtain

$$
G_{j}(z)=\sum_{n \geq 0}\left[-\frac{1}{2 \pi \mathrm{i}} \int_{\mathscr{C}} s^{n+k-j-1} \frac{p_{j}(s)}{P(s)} d s\right] \frac{z^{n}}{n !} .
$$

Then, (2.2) implies that

$$
b_{j}(k+n)=-\frac{1}{2 \pi \mathrm{i}} \int_{\mathscr{C}} s^{n+k-j-1} \frac{p_{j}(s)}{P(s)} d s, \quad 0 \leq j \leq k-1,
$$

and the result follows.

Remark 3.2. An alternative method for approximating the coefficients $b_{j}(n)$ is to write (3.1) as

$$
b_{j}(n)=-\frac{R^{n-j}}{2 \pi} \int_{0}^{2 \pi} \exp [\mathrm{i} t(n-j)] \frac{p_{j}\left(\mathrm{Re}^{\mathrm{i} t}\right)}{P\left(\mathrm{Re}^{\mathrm{i} t}\right)} d t
$$

with $R>\rho(A)$ and to compute the integral (3.5) numerically. This approach offers the advantage of avoiding the computation of the eigenvalues of $A$.

We now have all the necessary elements to establish our main theorem.

Theorem 3.3. Let

$$
\rho(A)=|\lambda|>\left|\lambda_{2}\right|>\cdots>\left|\lambda_{r}\right|
$$

be the eigenvalues of the matrix $A$, that is,

$$
P(x)=(x-\lambda)^{m}\left(x-\lambda_{2}\right)^{m_{2}} \cdots\left(x-\lambda_{r}\right)^{m_{r}}
$$

with $r \leq k$. Then,

$$
b_{j}(n) \sim-\lambda^{n-m-j} \frac{p_{j}(\lambda)}{P^{(m)}(\lambda)} n^{m-1} m, \quad n \longrightarrow \infty,
$$

where

$$
P^{(m)}(\lambda)=\left.\frac{d^{m} P}{d s^{m}}\right|_{s=\lambda} .
$$

Proof. To find an asymptotic approximation of (3.1), we will use a modified version of Darboux's method [14]. From (3.7) we have

$$
s^{n-j-1} \frac{p_{j}(s)}{P(s)} \sim \frac{p_{j}(\lambda)}{g(\lambda)} \frac{s^{n-j-1}}{(s-\lambda)^{m}}, \quad s \longrightarrow \lambda,
$$

where

$$
g(x)=\left(x-\lambda_{2}\right)^{m_{2}} \cdots\left(x-\lambda_{r}\right)^{m_{r}}
$$


Using the binomial theorem, we have

$$
s^{n-j-1}=\sum_{l=0}^{\infty}\left(\begin{array}{c}
n-j-1 \\
l
\end{array}\right)(s-\lambda)^{l} \lambda^{n-j-1-l} .
$$

Therefore,

$$
\begin{aligned}
b_{j}(n) & =-\frac{1}{2 \pi \mathrm{i}} \int_{\mathscr{C}} s^{n-j-1} \frac{p_{j}(s)}{P(s)} d s \sim-\frac{p_{j}(\lambda)}{g(\lambda)} \frac{1}{2 \pi \mathrm{i}} \int_{\mathscr{C}} \frac{s^{n-j-1}}{(s-\lambda)^{m}} d s \\
& =-\frac{p_{j}(\lambda)}{g(\lambda)}\left(\begin{array}{c}
n-j-1 \\
m-1
\end{array}\right) \lambda^{n-j-m} \sim-\frac{p_{j}(\lambda)}{g(\lambda)} \frac{n^{m-1}}{(m-1) !} \lambda^{n-j-m}, \quad n \longrightarrow \infty .
\end{aligned}
$$

To find the value of $g(\lambda)$, we use L'Hopital's theorem,

$$
g(\lambda)=\lim _{s \rightarrow \lambda} \frac{P(s)}{(s-\lambda)^{m}}=\lim _{s \rightarrow \lambda} \frac{P^{(m)}(s)}{m !}=\frac{P^{(m)}(\lambda)}{m !} .
$$

Replacing (3.14) in (3.13), we obtain (3.8).

Remark 3.4. Note that when $m=1$, we recover the leading term in (2.14).

If more than one eigenvalue has absolute value equal to the spectral radius of $A$, the asymptotic behavior of $b_{j}(n)$ can be obtained by adding the contributions from each eigenvalue. We state this formally in the following corollary.

Corollary 3.5. If

$$
\rho(A)=\left|\lambda_{1}\right|=\left|\lambda_{2}\right|=\cdots=\left|\lambda_{r}\right|,
$$

with respective multiplicities $m_{1}, m_{2}, \ldots, m_{r}$, then

$$
b_{j}(n) \sim-\sum_{l=1}^{r}\left(\lambda_{l}\right)^{n-m_{l}-j} \frac{p_{j}\left(\lambda_{l}\right)}{P^{\left(m_{l}\right)}\left(\lambda_{l}\right)} n^{m_{l}-1} m_{l}, \quad n \longrightarrow \infty .
$$

In the case when

$$
m_{1}=\cdots=m_{d}>m_{d+1} \geq m_{d+2} \geq \cdots \geq m_{r}
$$

one has

$$
b_{j}(n) \sim-n^{m_{1}-1} m_{1} \sum_{l=1}^{d}\left(\lambda_{l}\right)^{n-m_{1}-j} \frac{p_{j}\left(\lambda_{l}\right)}{P^{\left(m_{1}\right)}\left(\lambda_{l}\right)}, \quad n \longrightarrow \infty .
$$

Therefore, in the case of several eigenvalues located on the circle $|s|=\rho(A)$, the dominant term in (3.16) will correspond to the eigenvalue(s) with the greatest multiplicity.

Example 3.6. We now consider the case of more than one eigenvalue having absolute value equal to $\rho(A)$. Let

$$
P(x)=x^{4}+x^{3}-15 x^{2}-9 x+54=(x-2)(x-3)(x+3)^{2} .
$$


In this case, $\lambda_{1}=-3, m_{1}=2, \lambda_{2}=3, m_{2}=1$, and $k=4$. From (3.18), we have

$$
\begin{array}{ll}
b_{0}(n) \sim-\frac{1}{5} n(-3)^{n}, & b_{1}(n) \sim \frac{1}{10} n(-3)^{n}, \\
b_{2}(n) \sim \frac{1}{45} n(-3)^{n}, & b_{3}(n) \sim-\frac{1}{90} n(-3)^{n} .
\end{array}
$$

The exact values are

$$
\begin{aligned}
& b_{0}(n)=-\frac{1}{5} n(-3)^{n}+\frac{21}{50}(-3)^{n}-\frac{1}{2} 3^{n}+\frac{27}{25} 2^{n}, \\
& b_{1}(n)=\frac{1}{10} n(-3)^{n}-\frac{83}{300}(-3)^{n}-\frac{1}{12} 3^{n}+\frac{9}{25} 2^{n}, \\
& b_{2}(n)=\frac{1}{45} n(-3)^{n}+\frac{2}{225}(-3)^{n}+\frac{1}{9} 3^{n}-\frac{3}{25} 2^{n}, \\
& b_{3}(n)=-\frac{1}{90} n(-3)^{n}+\frac{11}{900}(-3)^{n}+\frac{1}{36} 3^{n}-\frac{1}{25} 2^{n} .
\end{aligned}
$$

As we observed before, the main contribution comes from the eigenvalue of maximum multiplicity, in this case $\lambda_{1}=-3$.

Example 3.7. Finally, let us consider the case of complex eigenvalues of multiplicity greater than one located on the circle $|s|=\rho(A)$. Let

$$
\begin{aligned}
P(x) & =x^{5}-9 x^{4}+34 x^{3}-66 x^{2}+65 x-25 \\
& =(x-1)[x-(2+i)]^{2}[x-(2-i)]^{2} .
\end{aligned}
$$

In this case, $\lambda_{1}=2+\mathrm{i}, m_{1}=2, \lambda_{2}=2-\mathrm{i}, m_{2}=2$, and $k=5$. From (3.18), we obtain

$$
\begin{gathered}
b_{0}(n) \sim \frac{1}{4}(\sqrt{5})^{n} n[\cos (\theta n)-7 \sin (\theta n)], \\
b_{1}(n) \sim-\frac{1}{10}(\sqrt{5})^{n} n[2 \cos (\theta n)-39 \sin (\theta n)], \\
b_{2}(n) \sim-\frac{1}{10}(\sqrt{5})^{n} n[2 \cos (\theta n)+31 \sin (\theta n)], \\
b_{3}(n) \sim \frac{1}{10}(\sqrt{5})^{n} n[2 \cos (\theta n)+11 \sin (\theta n)], \\
b_{4}(n) \sim-\frac{1}{20}(\sqrt{5})^{n} n[\cos (\theta n)+3 \sin (\theta n)],
\end{gathered}
$$

with

$$
\theta=\arctan \left(\frac{1}{2}\right)
$$


The exact values are

$$
\begin{gathered}
b_{0}(n)=\frac{1}{4}(\sqrt{5})^{n}[(n-21) \cos (\theta n)+(-7 n+22) \sin (\theta n)]+\frac{25}{4}, \\
b_{1}(n)=-\frac{1}{10}(\sqrt{5})^{n}[2(n-50) \cos (\theta n)+(-39 n+125) \sin (\theta n)]-10 \\
b_{2}(n)=-\frac{1}{10}(\sqrt{5})^{n}[(2 n+65) \cos (\theta n)+(31 n-100) \sin (\theta n)]+\frac{13}{2}, \\
b_{3}(n)=\frac{1}{10}(\sqrt{5})^{n}[2(n+10) \cos (\theta n)+(11 n-35) \sin (\theta n)]-2, \\
b_{4}(n)=-\frac{1}{20}(\sqrt{5})^{n}[(n+5) \cos (\theta n)+(3 n-10) \sin (\theta n)]+\frac{1}{4} .
\end{gathered}
$$

\section{Acknowledgment}

The author wishes to express his gratitude to the anonymous referees who provided him with invaluable suggestions and comments that greatly improved the first draft of the paper.

\section{References}

[1] R. Abu-Saris and W. Ahmad, Avoiding eigenvalues in computing matrix powers, The American Mathematical Monthly 112 (2005), no. 5, 450-454.

[2] N. Borovykh, D. Drissi, and M. N. Spijker, A bound on powers of linear operators, with relevance to numerical stability, Applied Mathematics Letters 15 (2002), no. 1, 47-53.

[3] N. Borovykh and M. N. Spijker, Resolvent conditions and bounds on the powers of matrices, with relevance to numerical stability of initial value problems, Journal of Computational and Applied Mathematics 125 (2000), no. 1-2, 41-56.

[4] W. E. Boyce and R. C. DiPrima, Elementary Differential Equations and Boundary Value Problems, John Wiley \& Sons, New York, 2005.

[5] S. Friedland and H. Schneider, The growth of powers of a nonnegative matrix, SIAM Journal on Algebraic and Discrete Methods 1 (1980), no. 2, 185-200.

[6] W. Gautschi, The asymptotic behaviour of powers of matrices, Duke Mathematical Journal 20 (1953), no. 1, 127-140.

[7] _ The asymptotic behaviour of powers of matrices. II, Duke Mathematical Journal 20 (1953), no. 3, 375-379.

[8] W. R. LePage, Complex Variables and the Laplace Transform for Engineers, Dover, New York, 1980.

[9] B. H. Lindqvist, Asymptotic properties of powers of nonnegative matrices, with applications, Linear Algebra and Its Applications 114/115 (1989), 555-588.

[10] K. H. Rosen, Elementary Number Theory and Its Applications, 4th ed., Addison-Wesley, Massachusetts, 2000.

[11] U. G. Rothblum, Expansions of sums of matrix powers, SIAM Review 23 (1981), no. 2, 143-164.

[12] P. Stavinoha, Convergence of $L_{p}$-norms of a matrix, Aplikace Matematiky 30 (1985), no. 5, 351360.

[13] N. M. Temme, Special Functions. An Introduction to the Classical Functions of Mathematical Physics, A Wiley-Interscience Publication, John Wiley \& Sons, New York, 1996. 


\section{Asymptotic analysis of powers of matrices}

[14] R. Wong, Asymptotic Approximations of Integrals, Classics in Applied Mathematics, vol. 34, SIAM, Pennsylvania, 2001.

[15] N. J. Young, Analytic programmes in matrix algebras, Proceedings of the London Mathematical Society. Third Series 36 (1978), no. 2, 226-242.

[16] Norms of powers of matrices with constrained spectra, Linear Algebra and Its Applications 23 (1979), 227-244.

Diego Dominici: Department of Mathematics, State University of New York at New Paltz,

75 S. Manheim Boulevard, Suite 9, New Paltz, NY 12561-2443, USA

E-mail address: dominicd@newpaltz.edu 


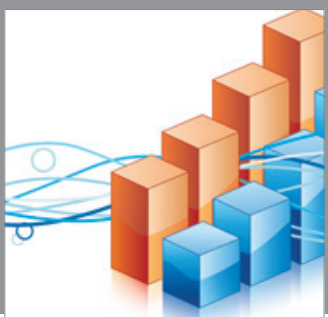

Advances in

Operations Research

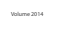

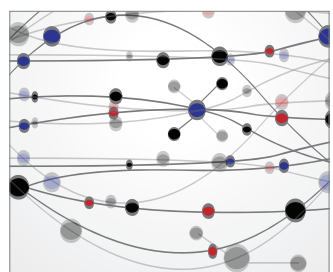

\section{The Scientific} World Journal
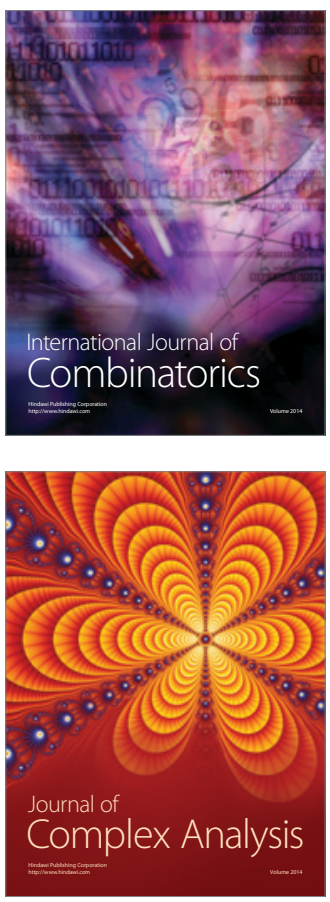

International Journal of

Mathematics and

Mathematical

Sciences
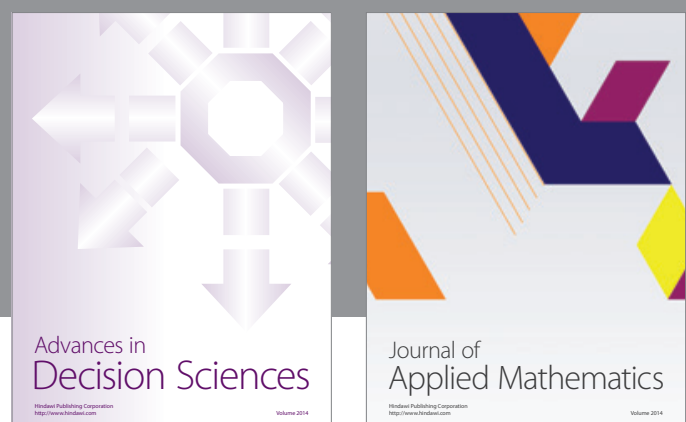

Journal of

Applied Mathematics
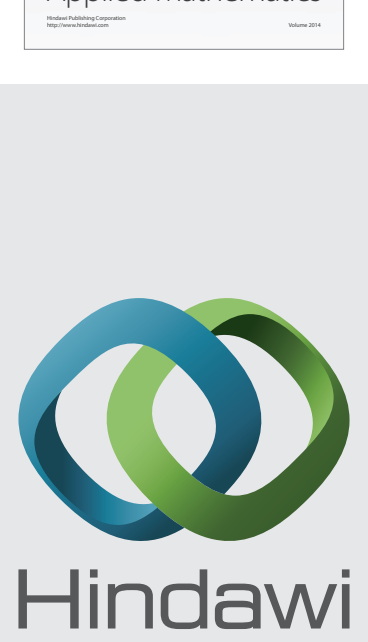

Submit your manuscripts at http://www.hindawi.com
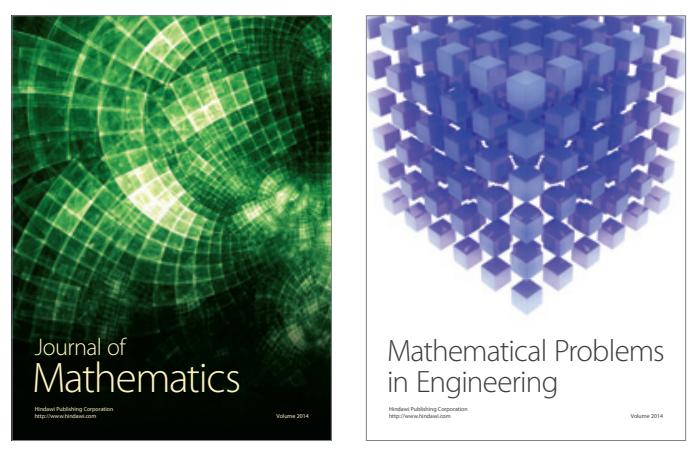

Mathematical Problems in Engineering
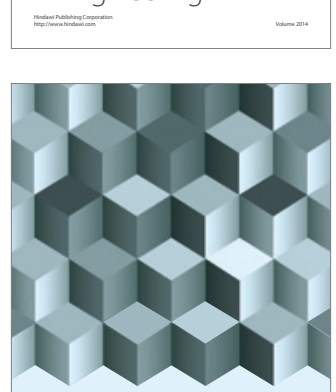

Journal of

Function Spaces
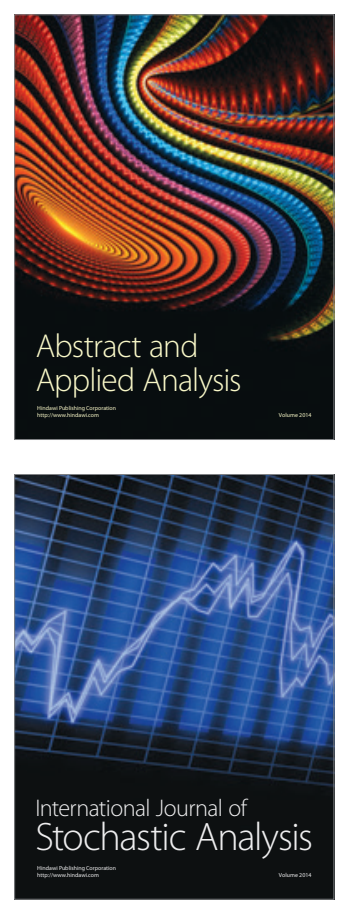

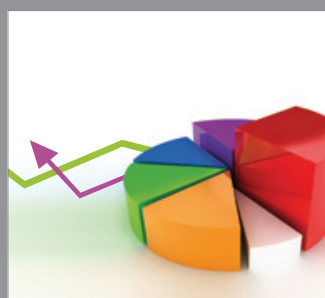

ournal of

Probability and Statistics

Promensencen
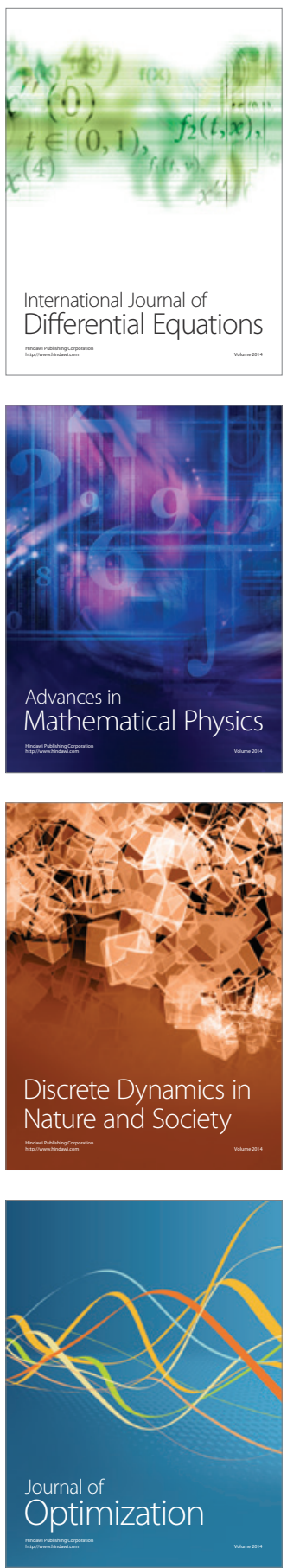